\title{
The identification of the star SPH 2 as a new WN4 star with strong C IV
}

\author{
Claudio Bastos Pereira \\ Departamento de Astronomia Galáctica, Observatório Nacional, \\ Rio de Janeiro, Brazil \\ Maria Auxiliadora Delgado Machado \\ Departamento de Astrofísica, Observatório Nacional, \\ Rio de Janeiro, Brazil
}

\begin{abstract}
We report on the spectroscopic observation of a new Wolf-Rayet star not already identified. This star is located in our Galaxy, in the Canis Majoris region and was found during a southern spectroscopic survey of stars previously identified as $\mathrm{H} \alpha$ emission objects. The star, SPH 2, after Schwartz, Persson \& Hamann (1990), shows the main characteristics of a WN star and a strong C IV $5808 \AA$ emission line typical of a WC star.
\end{abstract}

\section{Introduction}

In this work we present spectroscopic observations of the emission line star SPH 2, first recognized as $\mathrm{H} \alpha$ emission object after a prism-objective survey by Schwartz et al. 1990. This star was discovered as a new nitrogen Wolf-Rayet (WN) star in the framework of the spectroscopy survey of some $\mathrm{H} \alpha$ emission line objects in the southern hemisphere. Spectroscopic observations were performed using a Boller \& Chivens cassegrain spectrograph at $1.52 \mathrm{~m}$ ESO telescope at La Silla (Chile).

\section{Description of the spectrum}

The spectrum of SPH 2 shows the main nitrogen lines that characterizes a WN star. We can observe the presence of N IV $\lambda 4057 \AA$ and N v $\lambda 4603,4610 \AA$ used for WN classification, as well as the strong C IV at $5808 \AA$ characteristic of a WC spectrum. On the other hand, N III at $4640 \AA$ is absent. Smith et al. (1996), in their classification scheme for WN stars, use the He II 5411/He I 5875 ratio as the primary indicator of ionization. The presence of hydrogen is indicated by an oscillating Pickering decrement. Using these criteria, SPH 2, is classified as a WN4 without hydrogen. SPH 2 is listed as WR 7a in the VIIth Catalogue of Galactic Wolf-Rayet Stars (van der Hucht 1999 and in these Proceedings).

\section{Discussion and conclusions}

Conti \& Massey (1989) observed spectrophotometricaly nearly all galactic WolfRayet stars and in the Large Magellanic Cloud. They present a list of some WN 


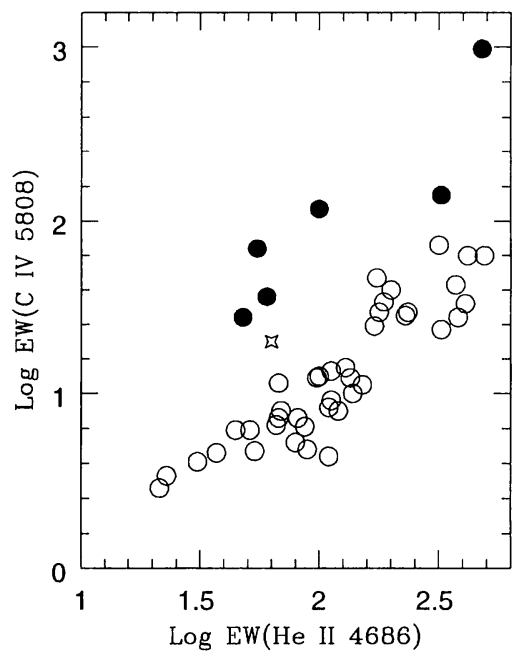

Figure 1. Log $E W$ He II $4686 \AA$ versus $\log E W \mathrm{C}$ IV $\lambda 5808 \AA$ as given by Conti \& Massey (1989). Empty circles represent galactic WN stars and full circles WN/WC stars. SPH 2 is the star in the middle of the diagram.

stars that shows C IV $\lambda 5808 \AA$ stronger than for a normal WN star. In their plot, the logarithm of the equivalent width of $\mathrm{C}$ IV $\lambda 5808 \AA$ versus the logarithm of the equivalent width of He II $\lambda 4686$ Asshows that there is clear difference between normal WN stars and WN/WC stars. Here, we reproduce their plot (Figure 1) where we include SPH2. We can see that SPH 2 occupies the locus halfway of the two groups. This would be an indication that SPH 2 lies in a transition stage between WN and WN/WC. This scenario will be further investigated in a subsequent paper, using an atmospheric model that includes nitrogen and carbon lines.

\section{References}

Conti, P.S., Massey, P. 1989, ApJ 337, 251

van der Hucht, K.A. 1999 in preparation

Smith, L.F., Shara, M.M, Moffat, A.F.J. 1996, MNRAS 281, 163

Schwartz, R.D., Persson, S.E., Hamann, F.W. 1990, AJ 100, 793 